\title{
The effect of exposure to lingual vibrotactile magnitude estimation on lingual vibrotactile magnitude production results
}

\author{
DONALD FUCCI \\ Ohio University, Athens, Ohio \\ LINDA PETROSINO \\ University of North Carolina, Chapel Hill, North Carolina \\ and \\ DANIEL HARRIS and ELISE M. McMATH \\ Ohio University, Athens, Ohio
}

\begin{abstract}
The purpose of the present investigation was to determine whether lingual vibrotactile magnitude production results were statistically equivalent between a group of 10 subjects (mean age $=21.1$ years) who performed magnitude estimation followed by magnitude production and a group of 10 subjects (mean age $=19.9$ years) who performed only magnitude production using the magnitude estimation numbers provided by the first group of subjects. Results showed statistical differences for magnitude production responses between the two groups. The data suggest that exposure to magnitude estimation procedure may have an influence on magnitude production results.
\end{abstract}

In 1983, a series of studies was initiated in which a psychophysical magnitude scaling technique was used in vibrotactile testing of the dorsal surface of the human tongue (Fucci, Harris, \& Petrosino, 1983a, 1983b; Fucci, Harris, \& Petrosino, 1984; Fucci \& Petrosino, 1983a, 1983b, 1983c; Fucci, Petrosino, \& Harris, 1983; Fucci, Petrosino, \& Harris, 1984; Petrosino, Fucci, \& Harris, 1984). The method of magnitude production was chosen for lingual vibrotactile scaling because it had been shown to be very stable and subjects tended to master it with ease (Stevens, 1961; Zwislocki \& Goodman, 1980). Pilot work also showed that magnitude production scaling could be managed on the tongue without serious restrictions being placed on stimulus intensity range and without subjects' becoming fatigued beyond the point of reliable testing.

The magnitude production procedure developed for lingual vibrotactile testing has received appropriate criticism due to the fact that the numbers used in scaling were derived by the experimenters rather than from the magnitude estimation responses of subjects. Fucci, Petrosino, and Harris, in an unpublished study, found that the numerical responses for lingual vibrotactile magnitude estimation for two large groups of subjects ( 37 subjects per group) were statistically equivalent. These experimenters proposed, therefore, that it might be possible to derive

Reprint requests should be addressed to Donald Fucci, Lindley 219. School of Hearing and Speech Sciences, Ohio University, Athens, $\mathrm{OH}$ 45701 . representative lingual vibrotactile magnitude estimation numbers from groups of subjects, and these could be used as the stimuli for lingual vibrotactile magnitude production experimentation. The development of a set of standardized lingual vibrotactile magnitude estimation numbers based on large numbers of subject responses would eliminate the need to perform the magnitude estimation task on subjects participating in studies in which magnitude production was the primary focus. A necessary step in the development of such a set of standardized magnitude estimation numbers would be to determine whether subject exposure to magnitude estimation has any influence on magnitude production results.

The purpose of the present study was to determine whether lingual vibrotactile magnitude production results were statistically equivalent between a group of subjects who performed magnitude estimation followed by magnitude production and a group of subjects who performed only magnitude production using the magnitude estimation numbers provided by the first group of subjects.

\section{METHOD}

\section{Subjects}

Two groups of 10 subjects each were selected randomly from students enrolled in introductory courses in hearing and speech sciences. Group 1 had an age range of 19 to 28 years (mean age $=21.1$ years). Group 2 had an age range of 18 to 22 years (mean age $=19.9$ years). All subjects had normal speech and hearing, and reported no known sensory and/or motor impairments. None of the subjects were experienced in vibrotactile testing or psychophysical scaling technique. 


\section{Apparatus}

The vibrotactile instrumentation consisted of a stimulus unit and a measurement unit. The stimulus unit was composed of a sine-wave generator, an experimenter-controlled variable attenuator, a subject-controlled variable attenuator, a rise/fall gate, two universal times, an audio amplifier, a power amplifier, a preamplifier, and an electromagnetic minivibrator with a probe-contactor extension. The pulsed vibratory signal generated had a frequency of $250 \mathrm{~Hz}$ with a $50 \%$ duty cycle (on $500 \mathrm{msec}$ and off $500 \mathrm{msec}$.) and a rise-and-decay time of $100 \mathrm{msec}$. The measurement unit consisted of an accelerometer, a cathode follower, a microphone amplifier, and a voltmeter. A narrow-band noise generator was used to present auditory masking at $70 \mathrm{~dB}$ HTL to the subjects in order to prevent them from hearing the vibratory stimulus. A detailed description of the vibrotactile equipment and procedures can be found in a review by Fucci, Petrosino, Wallace, and Small (1982).

\section{Procedure}

Each subject was seated in an adjustable chair and was asked to place his or her tongue against the underside of a rigidly mounted plastic disk. A hole in the center of the disk allowed the minivibrator probe-contactor extension to come into contact with the lingual dorsal surface. The contactor on the end of the probe had an area of $.128 \mathrm{~cm}^{2}$, and there was a 1-mm gap between the contactor and the plastic disk. The ascending method of limits was used to obtain vibrotactile thresholds of sensitivity for all subjects prior to initiation of the suprathreshold scaling task.

Group 1 participated in two test sessions. During the first session, the psychophysical method of magnitude estimation was used to obtain subjective magnitude functions for the lingual dorsal surface of each subject (Stevens, 1955). To minimize possible biases in the results, no reference standard was employed (Hellman \& Zwislocki, 1963). The subjects were requested to assign numbers to a randomly presented series of nine stimulus intensities, ranging from 2 to $40 \mathrm{~dB}$ sensation level (2, $6,10,14,20,24,30,34$, and $40 \mathrm{~dB}$ ). Each subject was instructed to "think of a number that matches the strength of the vibration." Whole numbers, decimals, and fractions were indicated as permissible selections (Zwislocki \& Goodman, 1980). The subjects were encouraged to be spontaneous in selecting numbers and to judge each stimulus without reference to those previously presented. The means of the subjects' responses over two runs of the nine stimulus intensities were accepted as the magnitude estimation numbers.

During the second test session, the psychophysical method of magnitude production was used to obtain subjective magnitude functions for the lingual dorsal surface of each of the Group 1 subjects (Stevens, 1958). The numbers presented to the subjects were the means of the subjective numbers provided by the same subjects during the magnitude estimation task $(0.4,0.7,1.4,2.0,4.0,5.5,9.6,11.0$, and 12.7). The subjects were instructed to "adjust the strength of the vibrotactile stimulus to match the number" presented by the experimenter. The subjectcontrolled attenuator consisted of a smooth unmarked knob (120-dB variable potentiometer) that provided no visual or mechanical cues. The experimenter was in control of a master attenuator that could be used to vary the amplitude of the stimulus delivered to the subject's attenuator. The means of two runs of the magnitude production values in millivolts were accepted as the resultant amplitudes.

Group 2 participated in a single test session. The psychophysical method of magnitude production was used to obtain subjective magnitude functions for the lingual dorsal surface of each subject (Stevens, 1958). The numbers presented to the subjects in Group 2 were the means of the magnitude estimation responses from Group 1 (the same numbers that had been presented to Group 1 for their magnitude production task). All other conditions were the same for the magnitude production tasks presented to Groups 1 and 2. The results of the magnitude production tasks for both groups of subjects were recorded in millivolts and converted to displacement in decibels re $1 \mu$ peak.

\section{RESULTS AND DISCUSSION}

The mean magnitude estimation responses for Group 1 and the mean magnitude production values for both groups
Table 1

Mean Magnitude Estimation Responses, Mean Magnitude Production Values (in Decibels SL), F Ratios, and Probability Values (Mean Magnitude Estimation Responses for Group 1 Were Used to Obtain the Mean Magnitude Production Values for Groups 1 and 2)

\begin{tabular}{|c|c|c|c|c|}
\hline $\begin{array}{c}\text { Group 1 } \\
\text { Mean } \\
\text { Magnitude } \\
\text { Estimation } \\
\text { Responses }\end{array}$ & $\begin{array}{c}\text { Group 1 } \\
\text { Mean } \\
\text { Magnitude } \\
\text { Production } \\
\text { Values } \\
\end{array}$ & $\begin{array}{c}\text { Group } 2 \\
\text { Mean } \\
\text { Magnitude } \\
\text { Production } \\
\text { Values } \\
\end{array}$ & F Ratios & $\begin{array}{c}\text { Probability } \\
\text { Values }\end{array}$ \\
\hline 0.4 & 7.67 & 4.64 & 1.67 & 0.21 \\
\hline 0.7 & 11.73 & 7.40 & 4.19 & 0.06 \\
\hline 1.4 & 16.31 & 10.69 & 8.27 & $0.01 *$ \\
\hline 2.0 & 19.68 & 13.68 & 8.48 & $0.01 *$ \\
\hline 4.0 & 23.47 & 17.45 & 7.51 & $0.01 *$ \\
\hline 5.5 & 25.99 & 20.91 & 5.14 & $0.04 *$ \\
\hline 9.6 & 28.28 & 24.69 & 3.41 & 0.08 \\
\hline 11.0 & 29.48 & 26.24 & 2.44 & 0.14 \\
\hline 12.7 & 30.99 & 28.39 & 1.47 & 0.24 \\
\hline
\end{tabular}

$*_{p}<.05$.

(in decibels SL) are shown in Table 1. The mean magnitude estimation responses from Group 1 were used to obtain the mean magnitude production values for Groups 1 and 2 . The magnitude production data were analyzed using a one-way (two-group) multivariate analysis of variance (Bock, 1975). The F ratios and probability values for both experimental groups are shown in Table 1. All statistical tests were conducted with an a priori alpha level of .05. Significant statistical differences were found for magnitude production between the two groups at four of the nine stimulus levels. The mean magnitude estimation responses that elicited group differences were 1.4, 2.0, 4.0 , and 5.5. The results of this study suggest that lingual vibrotactile magnitude production values for a group of subjects who perform magnitude estimation followed by magnitude production may be different from those for a group of subjects who perform only magnitude production using magnitude estimation numbers derived from another group of individuals.

It may be possible that the difference between the magnitude production results for the two groups was due to the exposure of Group 1 to the magnitude estimation task prior to magnitude production. Teghtsoonian and Teghtsoonian $(1971,1983)$ reported that a learning effect can take place in psychophysical scaling experimentation, and suggested that independent scaling responses require a minimum of $24 \mathrm{~h}$ between testing sessions. Even though there was at least one 1-week interval between the magnitude estimation and magnitude production performances of the subjects in Group 1 in the present study, they did receive the exposure to lingual vibrotactile magnitude estimation that was not given to Group 2 .

An alternative explanation for the difference in magnitude production results between the two groups might also be considered. Zwislocki and Goodman (1980) found that, in magnitude estimation and production procedures without designated moduli, people tend to scale sensation magnitudes on absolute, rather than ratio scales, and that 
the scales may be private and different for each individual. The difference between magnitude production results for Groups 1 and 2 of the present study could be viewed as supporting the concept that individual internal absolute scales were somewhat different between subjects in the two groups.

The statistically significant differences between lingual vibrotactile magnitude production results for the two groups of subjects in this study occurred at four of the nine stimulus levels utilized. Further research employing larger subject populations is warranted so that a more detailed analysis of the levels at which magnitude production differences occurred can be performed.

\section{REFERENCES}

Bock, R. D. (1975). Multivariate statistical methods in behavioral research. New York: McGraw-Hill.

Fucci, D., Harris, D., \& Petrosino, L. (1983a). The effect of contactor area on vibrotactile magnitude function exponents for the tongue and hand. Bulletin of the Psychonomic Society, 21, 400-402.

FuCCI, D., Harris, D., \& Petrosino, L. (1983b). Stability of magnitude production scales for lingual vibrotactile sensation. Journal of Perceptual and Motor Skills, 57, 499-504.

Fucci, D., Harris, D., \& Petrosino, L. (1984). Sensation magnitude scales for vibrotactile stimulation of the tongue and thenar eminence. Journal of Perceptual and Motor Skills, 58, 843-848.

FucCI, D., \& Petrosino, L. (1983a). Lingual vibrotactile sensation magnitudes: Comparison of suprathreshold responses for the tongue and hand. Journal of the Acoustical Society of America, 74, 351-353.

Fucci, D., \& PeTrosino, L. (1983b). Lingual vibrotactile sensation magnitudes: Comparison of suprathreshold responses for three different age ranges. Journal of Perceptual and Motor Skills, 57, 31-38.
FUCCI, D., \& PETrosino, L. (1983c). Lingual vibrotactile sensation magnitudes: Comparison of suprathreshold responses in men and women. Perception \& Psychophysics, 33, 93-95.

Fucci, D., Petrosino, L., \& HARRIS, D. (1983). Influence of scaling range on vibrotactile power function exponents for the tongue and hand. Journal of Perceptual and Motor Skills, 57, 642.

Fucci, D., Petrosino, L., \& HARris, D. (1984). Stability of magnitude production scales for oral and nonoral vibrotactile mechanoreceptive systems. Journal of Perceptual and Motor Skills, 58, 691-697.

Fucci, D., Petrosino, L., Wallace, D., \& Small, L. (1982). Modification of instrumentation for research on lingual vibrotactile sensitivity: Elimination of the tongue clamping procedure. Review of Scientific Instruments, 53, 1294-1296.

Hellman, R. P., \& ZWIsLocki, J. (1963). Monaural loudness function at $1000 \mathrm{cps}$ and interaural summation. Journal of the Acoustical Soiciety of America, 35, 856-865.

Petrosino, L., FuCCI, D., \& Harris, D. (1984). Threshold influences on vibrotactile magnitude production scaling. Bulletin of the Psychonomic Society, 22, 139-141.

StEVENS, S. S. (1955). The measurement of loudness. Journal of the Acoustical Society of America, 27, 815-820.

Stevens, S. S. (1958). Problems and methods of psychophysics. Psychological Bulletin, 54, 177-196.

Stevens, S. S. (1961). The psychophysics of sensory function. In W. A. Rosenblith (Ed.), Sensory communication. New York: Wiley.

Teghtsoonian, M., \& Teghtsoonian, R. (1971). How repeatable are Stevens's power law exponents for individual subjects? Perception \& Psychophysics, 10, 147-149.

Teghtsoonian, M., \& Teghtsoonian, R. (1983). Consistency of individual exponents in cross-modal matching. Perception \& Psychophysics, 33, 203-214.

ZWISLOCKI, J., \& Goodman, D. (1980). Absolute scaling of sensory magnitudes: A validation. Perception \& Psychophysics, 28, 28-38.

(Manuscript received for publication January 7, 1985.) 